\title{
Effects of a magnetic field and initial stress on reflection of SV-waves at a free surface with voids under gravity
}

\author{
${ }^{1}$ Department of Mathematics, COMSATS, Institute of Information, Park Road, Chakshahzad, Islamabad 44000, Pakistan, E- \\ mail:Aftab.khan@comsats.edu.pk \\ ${ }^{2}$ Department of Mathematics, Faculty of Science, South Valley University, Qena 83523, Egypt \\ ${ }^{3}$ Department of Mathematics, Faculty of Science, Taif University, Box 888, Taif, Saudi Arabia \\ ${ }^{4}$ Department of Mathematics, COMSATS, Institute of Information Technology, Wah Campus, Wah Cantt, Pakistan
}

\begin{abstract}
:
The present study deals with the reflection of SV-waves at a free surface in the presence of magnetic field, initial stress, voids and gravity. When an SV-wave incident on the free surface of an elastic half space, two damped Pwaves and an SV-wave are reflected. Among these waves, P-waves are only affected by magnetic fields whereas SV-waves are influenced by both, initial stress and magnetic fields. Effect of gravity is negligible whereas voids played a significant role. These observations can be helpful for seismology and earthquake sciences.
\end{abstract}

Keywords: initial stress, gravity, P-wave, reflection, SV-wave, voids

DOI: $10.1515 / \mathrm{jmbm}-2018-0002$

\section{Introduction}

Seismic wave propagation has gained much attention in the last few years for study, because of their relevance to many difference applications in several branches of science and technology, such as earthquake science, geophysics and optics. The general equation of reflection and refraction of elastic waves at a plane half-space was first derived by Knott [1] and subsequently developed by Jefferys [2], Gutenberg [3] and many others but they did not take initial stress in the solid half-space, into account. Generally, several media are initially stressed due to many physical causes. The earth is also an initially stressed medium. Biot [4] has proved that the initial stress has significant effect on the propagation of elastic waves. Latterly, other authors like Dey and Addy [5] included the effect of initial stress on the reflection of waves at the solid half-space.

In the classical theory of elasticity, voids are an important generalization. Nunziato and Cowin [6] and Cowin and Nunziato [7] discussed the theory in elastic media with voids. Puri and Cowin [8] studied the effects of voids on plane waves in linear elastic media and it is evident that pure shear waves remain unaffected by the presence of pores. Chandrasekharaiah [9] and [10] discussed the effects of voids on propagation of plane and surface waves. Abo-Dahab [11] and Atwa et al. [12] investigated the propagation of P-waves from stress-free surface elastic half-space with voids.

Wave propagation in the presence of initial stress and voids is of great significance in numerous fields such as earthquake engineering, soil dynamics, composite materials in aeronautics, astronautics and nuclear reactors, etc. The investigation of wave propagation in an isotropic generalized solid in the presence of initial stress, voids and a magnetic field under the influence of gravity yields information about the existence of new or modified waves. This information may be efficacious for experimental seismologists in correcting earthquake estimation.

This paper investigates the problem of the reflection of a plane SV-wave incident on an initially stressed, homogenous, isotropic solid half-space with voids under the influence of gravity and magnetic field. In this study, firstly, the stress-strain relations with incremental isotropy introduced by Biot [4] are used, and then a modified void equation was used. Furthermore, the governing equations are solved analytically for two-dimensional motions in the $x_{1} x_{2}$-plane to obtain the expressions for displacement potentials and velocities. Applying the free surface boundary conditions, expressions for the reflection coefficients are derived. Numerical computation for a chosen material is also discussed. 


\section{Formulation and solution of the problem}

Governing equations with initial stress and a magnetic field under the influence of gravity in a homogenous elastic medium are as follows:

i. The equations of motion:

$$
\tau_{i j, j}+\vec{F}_{i}+\vec{G}_{i}=\rho \ddot{u}_{i}
$$

where $\vec{F}=\mu_{0}(\underline{J} \times \underline{H}), \vec{G}_{i}=\left(\rho g \varepsilon_{3 j k} u_{j, k}\right) \delta_{i j}$, In component form, equation of motion becomes,

$$
\begin{aligned}
& \tau_{11,1}+\tau_{12,2}+F_{1}-\rho g u_{2,1}=\rho \ddot{u}_{1}, \\
& \tau_{21,1}+\tau_{22,2}+F_{2}+\rho g u_{1,1}=\rho \ddot{u}_{1} .
\end{aligned}
$$

ii. The equation for voids:

$$
\alpha \varphi_{, i i}-\omega_{0} \varphi-v \dot{\varphi}-\beta u_{i, i}=\rho \kappa \ddot{\varphi},
$$

iii. Constitutive relations:

$$
\tau_{i j}=-P\left(\delta_{i j}+\omega_{i j}\right)+\lambda \varepsilon_{k k} \delta_{i j}+2 \mu \varepsilon_{i j}+\beta \delta_{i j} \varphi, \text { where } \varepsilon_{i j}=\frac{1}{2}\left(u_{i, j}+u_{j, i}\right), \omega_{i j}=\frac{1}{2}\left(u_{i . j}-u_{j, i}\right) .
$$

We take the linearized Maxwell equations governing the electromagnetic field for a perfectly conducting medium as follows:

$$
\begin{aligned}
& \operatorname{Curl} \underline{H}=J \times \varepsilon_{o} \dot{E}, \\
& \operatorname{Cur} \underline{\underline{E}}=-\mu_{o} \dot{H}, \\
& \operatorname{Div} \underline{H}=0, \operatorname{Div} \underline{E}=0, \\
& \underline{E}=\mu_{o}(\underline{\dot{u}} \times \underline{H}), \\
& \underline{h}=(\underline{u} \times \underline{H}),
\end{aligned}
$$

\footnotetext{
where $H=H_{0}+h, h$ is the induced magnetic force and $\varepsilon_{0}$ is the electric permeability. Initial applied magnetic field $H_{0}=\left(0,0, \bar{H}_{0}\right)$, i.e. taken along the $x_{3}$ axis and the material lies in $x_{1} x_{2}$ plane.

Thus, $H=H_{0}+h=\left(h_{1}, h_{2}, h_{3}+H_{0}\right)$.

Then the magnetic force is as follows

$$
\vec{F}=\mu_{0} H_{0}^{2}\left(e_{, 1}-\varepsilon_{0} \mu_{0} \ddot{u}_{1}, e_{, 2}-\varepsilon_{0} \mu_{0} \ddot{u}_{2}, 0\right) \text { and } \bar{h}\left(x_{1}, x_{2}, x_{3}\right)=(0,0,-e),
$$

where $e=u_{1.1}+u_{2,2}$.

In these equations, $F_{i}$ represents the magnetic force, $J$ is the current density, $\underline{H}$ is the magnetic field vector and $\mu_{0}$ is the magnetic permeability. $\varphi$ is the so-called volume fraction field. $\alpha, \beta, \omega_{0}, \nu$ and $\kappa$ are new material constants characterizing the presence of voids. Where $\varepsilon_{i j k}$ is the Levi-Civita tensor, $\tau_{i j}$ are the components of stress, $\rho$ is the mass density and $u_{i}$ is the displacement vector. $\lambda$ and $\mu$ are elastic constants and $u_{i}$ is the displacement component. A comma followed by the index shows a partial derivative with respect to a coordinate. Also, Einstein's summation convention over repeated indexes is used.

Here we consider a half space which is a homogenous and isotropic elastic solid. The $x_{1} x_{2}$ half-space is chosen to coincide with the free surface with initial compressive stress $P$ in the $x_{1}$ direction. A plane wave incident at " 0 " on the boundary surface in the $x_{1} x_{2}$ plane at $x_{2}=0$, making an angle $\theta_{0}$, with the normal to the boundary as shown in Figure 1.
} 


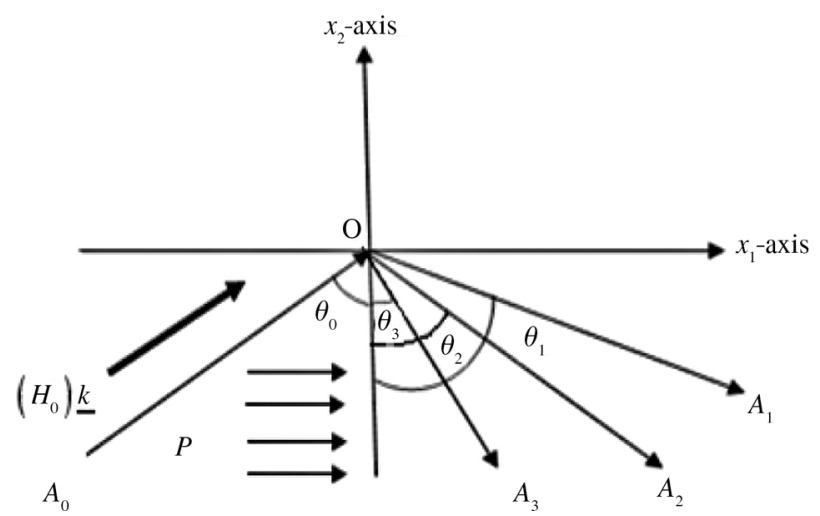

Figure 1: Schematic of the problem.

Substituting equations (3) into (1), we have

$$
\begin{aligned}
& \left(\begin{array}{c}
\lambda+2 \mu \\
+\mu_{o} H_{0}^{2}
\end{array}\right) u_{1,11}+\left(\begin{array}{c}
\lambda+\mu+\frac{\mathrm{P}}{2} \\
+\mu_{0} H_{0}^{2}
\end{array}\right) u_{2,12}+\left(\mu-\frac{\mathrm{P}}{2}\right) u_{1,22}-\rho g u_{2,1}=\left(\begin{array}{c}
\rho \\
+\mu_{o}{ }^{2} \varepsilon_{0} H_{0}{ }^{2}
\end{array}\right) \ddot{u}_{1}-\beta \varphi_{, 1} \\
& \left(\begin{array}{c}
\lambda+2 \mu \\
+\mu_{0} H_{0}^{2}
\end{array}\right) u_{2,22}+\left(\begin{array}{c}
\lambda+\mu+\frac{\mathrm{P}}{2} \\
+\mu_{o} H_{0}{ }^{2}
\end{array}\right) u_{1,12}+\left(\mu-\frac{\mathrm{P}}{2}\right) u_{2,11}+\rho g u_{1,1}=\left(\begin{array}{c}
\rho \\
+\mu_{o}{ }^{2} \varepsilon_{0} H_{0}{ }^{2}
\end{array}\right) \ddot{u}_{2}-\beta \varphi_{, 2} .
\end{aligned}
$$

The modified voids equation is as follows:

$$
\alpha\left(\varphi_{, 11}+\varphi_{, 22}\right)-\omega_{0} \varphi-v \dot{\varphi}-\beta\left(u_{1,1}+u_{2,2}\right)=\rho \kappa \ddot{\varphi}
$$

Using Helmholtz's theorem, the displacement vector $\vec{u}$ can be written in the displacement potentials $\phi$ and $\psi$ form

$$
\vec{u}=\operatorname{Grad} \phi+\operatorname{Curl} \psi
$$

which reduces to

$$
\begin{aligned}
u_{1}=\phi_{, 1}+\psi_{, 2} \text { and } u_{2} & =\phi_{, 2}-\psi_{, 1} \\
\psi & =\psi(0,0,1) .
\end{aligned}
$$

By using (8 and 9) into equation (5), we have

$$
\gamma_{1} \nabla^{2} \phi=\gamma_{2} \frac{\partial^{2} \phi}{\partial t^{2}}-\rho g \frac{\partial \psi}{\partial x_{1}}-\beta \varphi .
$$

By using (8 and 9) in equation (6), we have

$$
\gamma_{3} \nabla^{2} \psi=\gamma_{2} \frac{\partial^{2} \psi}{\partial t^{2}}+\rho g \frac{\partial \phi}{\partial x_{1}}
$$

where

$$
\gamma_{1}=\lambda+2 \mu+\mu_{o} H_{0}^{2}, \gamma_{2}=\rho+\mu_{o}{ }^{2} \varepsilon_{0} H_{0}{ }^{2}, \gamma_{3}=\mu-\frac{1}{2} P
$$

Substituting (8 and 9) into (7), we have

$$
\alpha\left(\nabla^{2} \varphi\right)=\omega_{0} \varphi+v \dot{\varphi}+\beta\left(\nabla^{2} \phi\right)+\rho \kappa \ddot{\varphi}
$$


The solutions of equations (10-13) can be taken as:

$$
\begin{aligned}
& \phi\left(x_{1}, x_{2}, t\right)=\phi_{0} \exp \left[i k\left(\sin \theta x_{1}+\cos \theta x_{2}-c t\right)\right], \\
& \psi\left(x_{1}, x_{2}, t\right)=\psi_{0} \exp \left[i k\left(\sin \theta x_{1}+\cos \theta x_{2}-c t\right)\right], \\
& \varphi\left(x_{1}, x_{2}, t\right)=\varphi_{0} \exp \left[i k\left(\sin \theta x_{1}+\cos \theta x_{2}-c t\right)\right],
\end{aligned}
$$

Using (14-16) in (10), (11) and (13), we have

$$
\begin{gathered}
k^{2}\left(\gamma_{1}-c^{2} \gamma_{2}\right) \phi_{0}-\beta \varphi_{0}-i \rho g k \sin \theta \psi_{0}=0, \\
(2 i k c \rho \Omega+i \rho g k \sin \theta) \phi_{0}-k\left(\gamma_{3}-c^{2} \gamma_{2}\right) \psi_{0}=0 \\
\beta k^{2} \phi_{0}+\left(k^{2}\left(\rho \kappa c^{2}-\alpha\right)+i v k c-\omega_{0}\right) \varphi_{0}=0 .
\end{gathered}
$$

Eliminating $\phi_{0}, \psi_{0}$, and $\varphi_{0}$ from equations (17-19), we have

$$
C_{1} V^{3}+C_{2} V^{2}+C_{3} V+C_{4}=0,
$$

where

$$
\begin{aligned}
& V=c^{2}, \\
& C_{1}=-\rho \kappa \gamma_{2}^{2} k^{5} \\
& C_{2}=\rho \kappa k^{5} \gamma_{2}\left(\gamma_{1}+\gamma_{3}\right)+k^{3} \gamma_{2}^{2}\left(\alpha k^{2}+\omega_{0}\right) \\
& C_{3}=\beta^{2} k^{3} \gamma_{2}+-k^{5} \gamma_{1} \gamma_{3} \rho \kappa+\rho^{3} \kappa k\left(g^{2} k^{3} \operatorname{Sin}^{2} \theta\right)-k^{3} \gamma_{2}\left(\gamma_{1}+\gamma_{3}\right)\left(\alpha k^{2}+\omega_{0}\right) \\
& C_{4}=-\left(\alpha k^{2}+\omega_{0}\right)\left\{\rho^{2}\left(g^{2} k^{2} \operatorname{Sin}^{2} \theta\right)-k^{3} \gamma_{1} \gamma_{3}\right\}-k^{4} \gamma_{3} \beta^{2}
\end{aligned}
$$

It is obvious from (20) that it has three roots which corresponds three velocities for reflected waves.

\section{Reflection coefficients}

There are three reflected waves, P-wave, SV-wave and a wave due to voids. Thus, if a rotational wave falls on boundary $x_{2}=0$ from the solid half space we have one reflected rotational wave and two reflected compressional waves traveling with two different velocities. Accordingly, if the normal wave of the incident rotational wave makes an angle $\theta_{0}$ with the positive $x_{2}$-axis and those of reflected SV-, P- and void-waves make angles $\theta_{1}, \theta_{2}$ and $\theta_{3}$ with the same direction. The displacement potential and the volume fraction field take the following forms:

$$
\begin{gathered}
\psi=A_{0} \exp \left[i\left\{k_{0}\left(x_{1} \operatorname{Sin} \theta_{0}+x_{2} \operatorname{Cos} \theta_{0}\right)-\omega t\right\}\right]+\sum_{j=1}^{3} A_{j} \exp \left[i\left(k_{j}\left(x_{1} \operatorname{Sin} \theta_{j}-x_{2} \operatorname{Cos} \theta_{j}\right)-\omega t\right)\right], \\
\varphi=\zeta_{0} A_{0} \exp \left[i\left\{k_{0}\left(x_{1} \operatorname{Sin} \theta_{0}+x_{2} \operatorname{Cos} \theta_{0}\right)-\omega t\right\}\right]+\sum_{j=1}^{3} \zeta_{j} A_{j} \exp \left[i\left\{k_{j}\left(x_{1} \operatorname{Sin} \theta_{j}-x_{2} \operatorname{Cos} \theta_{j}\right)-\omega t\right\}\right], \\
\varphi=\eta_{0} A_{0} \exp \left[i\left\{k_{0}\left(x_{1} \operatorname{Sin} \theta_{0}+x_{2} \operatorname{Cos} \theta_{0}\right)-\omega t\right\}\right]+\sum_{j=1}^{3} \eta_{j} A_{j} \exp \left[i\left\{k_{j}\left(x_{1} \operatorname{Sin} \theta_{j}-x_{2} \operatorname{Cos} \theta_{j}\right)-\omega t\right\}\right],
\end{gathered}
$$

where

$$
\zeta_{j}=\frac{k\left(\gamma_{3}-\frac{\rho \Omega^{2}}{k^{2}}-\gamma_{2} c_{j}^{2}\right)}{2 i \Omega \rho c_{i}} \text { and } \eta_{j}=\frac{-\beta k_{j}^{2} \zeta_{j}}{k_{j}^{2}\left(\rho \kappa c_{j}^{2}-\alpha\right)+i v k c_{j}-\omega_{0}}
$$

where, $A_{0}$ is the amplitude of the incident P-wave and $A_{1}, A_{2}$ and $A_{3}$ are the amplitudes of reflected SV-, P- and wave due to voids, respectively. 


\section{Boundary conditions}

As the boundary at $x_{2}=0$ is adjacent to a vacuum, it is free from surface tractions, therefore

$$
\tau_{i 2}+P\left(\delta_{i 2}+\omega_{i 2}\right)+\bar{\tau}_{i 2}=0, \quad \text { at } x_{2}=0
$$

where, Maxwell's stresses are as follows:

$$
\begin{gathered}
\bar{\tau}_{i j}=\mu_{0} H_{0}\left[H_{i} h_{j}+H_{j} h_{i}-H_{k} h_{k} \delta_{i j}\right], \\
\tau_{12}+P \omega_{12}=0, \quad \text { at } x_{2}=0 \quad \bar{\tau}_{12}=0 \\
\tau_{22}+P+\bar{\tau}_{22}=0, \quad \text { at } x_{2}=0
\end{gathered}
$$

It is also assumed that there is no change in volume traction, $\varphi$, along the $x_{2}$-direction, thus

$$
\frac{\partial \varphi}{\partial x_{2}}=0, \quad \text { at } x_{2}=0
$$

Using equations (21-23) in (24-26), we get

$$
\sum A_{i j} Z_{j}=D_{i,} \quad(i, j=1,2,3)
$$

where

$$
\begin{aligned}
A_{1 j} & =\left[\operatorname{Cos} 2 \theta_{j}-\zeta_{j} \operatorname{Sin} 2 \theta_{j}\right]\left(\frac{k_{j}}{k_{0}}\right)^{2}, \\
A_{2 j} & =\left[\lambda \zeta_{j}+\mu\left(2 \zeta_{j} \operatorname{Cos}^{2} \theta_{j}-\operatorname{Sin} 2 \theta_{j}\right)+\mu_{0} H_{0}{ }^{2}+\beta \frac{\eta_{j}}{k_{j}^{2}}\right]\left(\frac{k_{j}}{k_{0}}\right)^{2}, \\
A_{3 j} & =\eta_{j} \operatorname{Cos} \theta_{j}\left(\frac{k_{j}}{k_{0}}\right),
\end{aligned}
$$

and

$$
\begin{aligned}
& D_{1}=\left[\operatorname{Cos} 2 \theta_{0}-\zeta_{0} \operatorname{Sin} 2 \theta_{0}\right] \\
& D_{2}=-\left[\lambda \zeta_{0}+\mu\left(2 \zeta_{0} \operatorname{Cos}^{2} \theta_{0}-\operatorname{Sin} 2 \theta_{0}\right)+\mu_{0} H_{0}^{2}+\beta \frac{\eta_{0}}{k_{0}^{2}}\right] \\
& D_{3}=\eta_{0} \operatorname{Cos} \theta_{0} \\
& Z_{1}=R_{C 1}=\frac{A_{1}}{A_{0}}, Z_{2}=R_{C 2}=\frac{A_{2}}{A_{0}}, Z_{3}=R_{C 3}=\frac{A_{3}}{A_{0}} .
\end{aligned}
$$

\section{Numerical results and discussion}

With the view of computational work, we take the following physical constants.

$$
\begin{aligned}
& \lambda=5.65 \times 10^{10} \mathrm{Nm}^{-2}, \mu=2.46 \times 10^{10} \mathrm{Nm}^{-2}, \rho=2.66 \times 10^{3} \mathrm{kgm}^{-3}, \\
& \alpha=-1.28 \times 10^{10} \mathrm{Nm}^{-2}, \beta=220.90 \times 10^{10} \mathrm{Nm}^{-2} .
\end{aligned}
$$

Using these values, the modulus of the reflection coefficients for the SV-wave and P-wave have been calculated for different angles of incidence.

We plotted a graph to show the variation of reflection coefficient $R_{C 1}$ of the P-wave with the variation of magnetic field $H_{0}$ with respect to the angle of incidence $\theta_{0}$ keeping $\kappa, \omega, \alpha, \Omega, P, \varepsilon_{0}, \omega_{0}$ constant (see Figure 
$2 \mathrm{~A})$. From this graph, it can be seen, that $R_{C 1}$ decreases with the increase in $\theta_{0}$. It is also observed that reflection coefficient decreases as $H_{0}$ increases. Figure $2 \mathrm{~B}$ shows that reflection coefficient $R_{\mathrm{C} 2}$ of the $\mathrm{SV}$-wave is unaffected by the magnetic field, whereas Figure $2 \mathrm{C}$ shows that reflection coefficient decreases as $H_{0}$ increases. There will be no reflection for the angle of incidence $\theta_{0}=\frac{\pi}{2}$ and reflection coefficients have a maximum value as the angle of incidence $\theta_{0} \rightarrow 0$ and reflection does not exist at $\theta_{0}=0$.
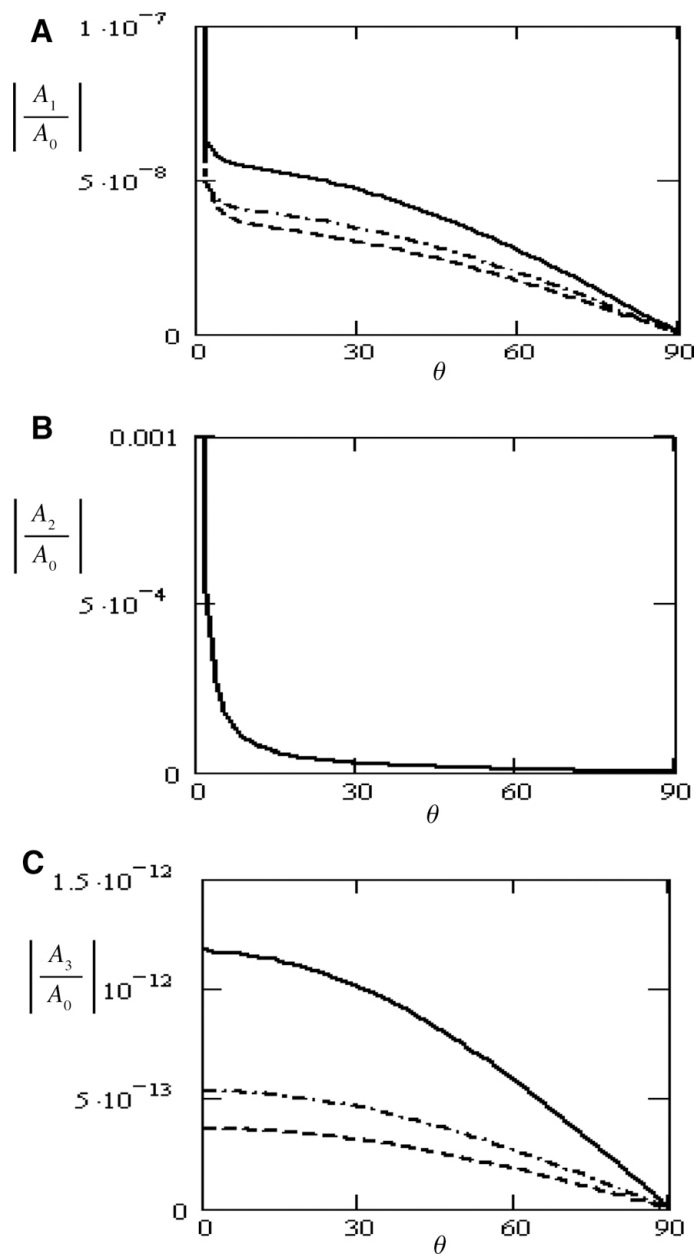

Figure 2: Variations of a magnetic field $H=0.1 \_, 0.3-.-., 0.5---$ on the magnitude of amplitude ratios with respect to the angle of incidence.

Furthermore, in Figure 3A we show that the variation of reflection coefficient $R_{C 1}$ of the P-wave with the variation of initial stresses $P$ with respect to the angle of incidence $\theta_{0}$ keeping $\kappa, \omega, \alpha, H_{0}, \Omega, \varepsilon_{0}, \omega_{0}$ as constants. From this figure, it can be observed that $R_{C 1}$ decreases with the increase in $\theta_{0}$. It is also shown that reflection coefficient increases as $P$ increases. Figure 3B shows that reflection coefficient $R_{\mathrm{C} 2}$ of the SV-wave is unaffected by initial stresses, whereas Figure $3 C$ shows that reflection coefficient increases as $P$ increases. There will be no reflection for angle of incidence $\theta_{0}=\frac{\pi}{2}$ and reflection coefficients are maximum for angle of incidence $\theta_{0}=0$. 


\section{A}

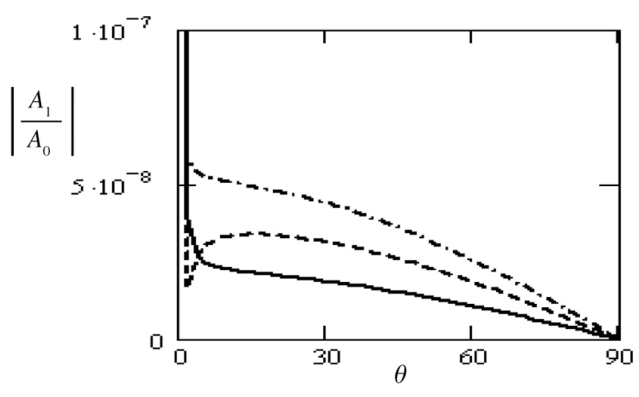

B
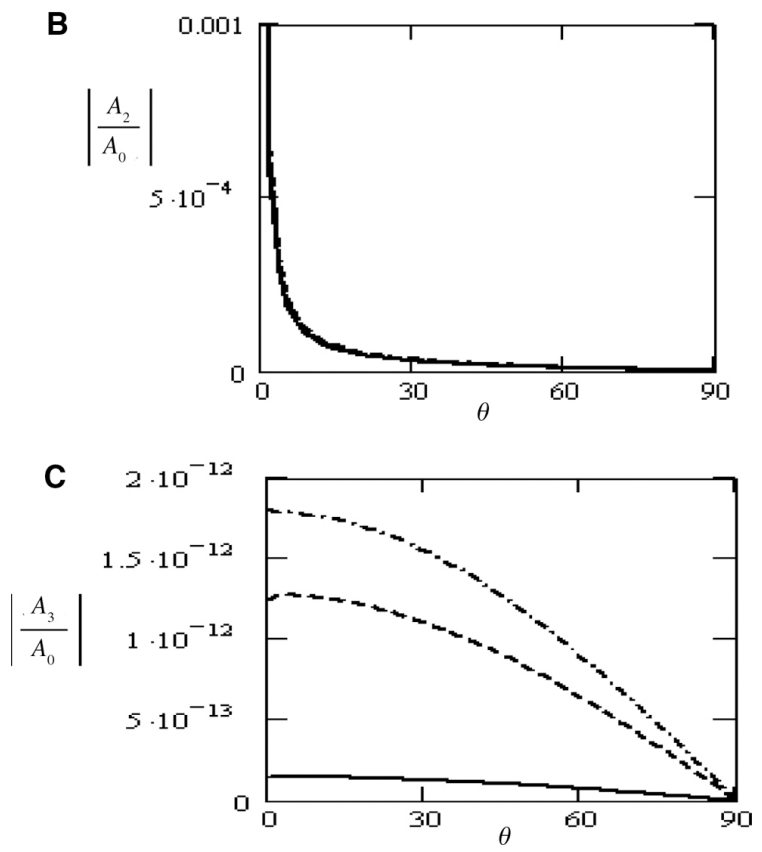

Figure 3: Variations of initial stress $P=10^{10}$ $2\left(10^{10}\right)-.-., 3\left(10^{10}\right)---$ on the magnitude of amplitude ratios with respect to the angle of incidence.

In Figure $4 \mathrm{~A}$, we show the variation of reflection coefficient $R_{C 1}$ of the P-wave with the variation of electric field $\varepsilon_{0}$ with respect to the angle of incidence $\theta_{0}$ keeping $\kappa, \omega, \alpha, H_{0} \Omega, P, \omega_{0}$ constant. It is observed that $R_{C 1}$ decreases with the increase in $\varepsilon_{0}$. It is also noted that reflection coefficient increases as $\varepsilon_{0}$ increases. In Figure $4 \mathrm{~A}$ and $\mathrm{B}$, we plotted these graphs to show that reflection coefficient $R_{\mathrm{C} 2}$ of the SV-wave is unaffected by an electric field, whereas, Figure $4 \mathrm{C}$ shows that reflection coefficient increases as $\varepsilon_{0}$ increases. There will be no reflection for angle of incidence $\theta_{0}=\frac{\pi}{2}$ and reflection coefficients have maximum value near the angle of incidence $\theta_{0}=$ 0 and reflection does not exist at $\theta_{0}=0$. 

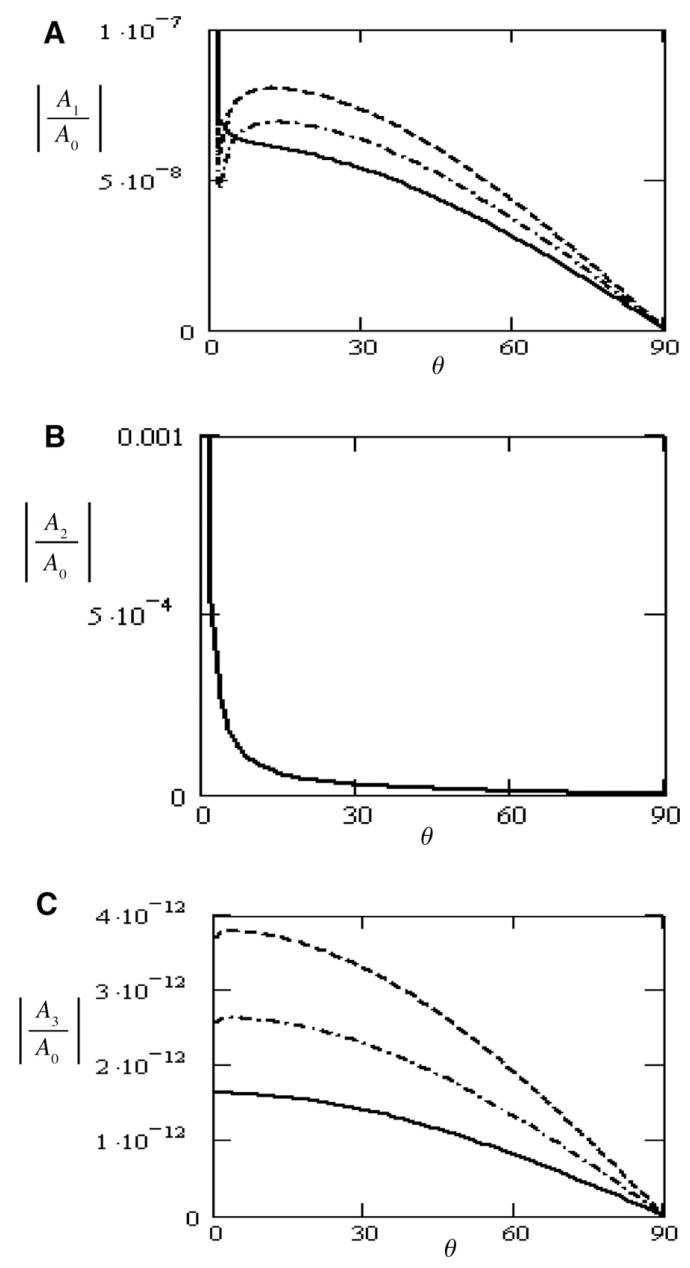

Figure 4: Variations of electric field $\varepsilon=0.01$ 0.02-.-., 0.04- - on the magnitude of amplitude ratios with respect to the angle of incidence.

\section{Conclusion}

The reflection of SV-waves on a free surface under a magnetic field, initial stress and electric field with voids was studied. In addition, expressions for reflection coefficients for P-waves, SV-waves and waves due to voids are derived. Numerical results for a chosen material, aluminum, for different parameters are given and illustrated graphically. It is observed that a magnetic field, initial stress and electric field significantly affect the reflection coefficients. In the absence of voids, the results reduce to well-known isotropic medium.

\section{References}

[1] Knott CG. Phil. Mag. 1899, 48, 64-97.

[2] Jeffreys H. Proc. Cambridge Phil. Soc. 1930, 26, 101-106.

[3] Gutenberg R. Bult. Seis. Soc. Amer. 1944, 34, 85-102.

[4] Biot MA. Mechanics of Incremental Deformations. John Wiley and Sons, Inc.: New York, 1965.

[5] Dey S, Addy SK. Int. J. Nonlinear Mechanics. Pergamon Press. 1977, 12, 372-381.

[6] Nunziato JW, Cowin SC. Arch. Rational. Mech. Anal. 1979, 72 (2), 175-201.

[7] Cowin SC, Nunziato JW. J. Elasticity. 1983, 13 (2), 125-147.

[8] Puri P, Cowin SC. J. Elasticity. 1985, 15 (2), 167-183.

[9] Chandrasekharaiah DS. Int. J. Eng. Sci. 1987, 25 (2), 205-211.

[10] Chandrasekharaiah DS. Int. J. Eng. Sci. 1987, 25 (5), 591-596.

[11] Abo-Dahab SM. Appl. Math. Model. 2010, 34 (7), 1798-1806.

[12] Atwa SY, Nazeer M, Adnan ], Rehman N. Eur. Phys. J. Plus 2017, 132, 1-11. 